\title{
Steady-state behavior of finite compliant journal bearing using a piezoviscous polar fluid as lubricant
}

\author{
Ahcene Mouassa $^{1}$, Hamid Boucherit ${ }^{1}$, Benyebka Bou-SaÏD ${ }^{2, a}$, Mustapha Lahmar ${ }^{1}$, \\ Hamza Bensouilah ${ }^{1}$ and Salah Ellagoune ${ }^{1}$ \\ 1 Laboratoire de Mécanique et Structures, Université 8 mai 1945, BP 401, Guelma, Algeria \\ 2 Université de Lyon, CNRS INSA-Lyon, LaMCoS, UMR5259, 69621 Villeurbanne, France
}

Received 26 December 2014, Accepted 30 May 2015

\begin{abstract}
The proposed work is concerned with the theoretical and numerical investigation of the lubricant rheology effects on the steady-state behavior of a plain finite compliant journal bearing operating under isothermal conditions. In the present investigation, the couple-stresses due to the presence of improving viscosity index (VI) additives, the viscosity-pressure (piezoviscosity effect) as well as the density-pressure (compressibility effect) variations are considered. The hydrodynamic lubrication theory is based on the V.K. Stokes micro-continuum mechanics which takes into account the size of macro-molecular chains added to the basic oil. The Barus and Dowson-Higginson laws were used to express the viscosity-pressure and density-pressure variations. Using the classical assumptions of lubrication, a modified Reynolds' equation is derived and solved numerically by the finite difference method. The displacement field at the fluid film bearing liner interface due to pressure forces is determined using the elastic thin liner model. The proposed work is concerned with the theoretical and numerical investigation of the lubricant rheology effects on the steady-state behavior of a plain finite compliant (elastic liner) journal bearing operating under isothermal conditions and laminar flow. The obtained results show that the couple-stresses have significant effects on the hydrodynamic performance characteristics such as the pressure field, the carrying capacity, the attitude angle and friction number especially when the viscosity-pressure variation is considered. Moreover, it is also shown that the compressibility of lubricant doesn't affect the hydrodynamic characteristics.
\end{abstract}

Key words: Hydrodynamic journal bearings / couple stress fluids / non-newtonian lubricants / Reynolds' equation / viscosity additives

\section{Introduction}

Hydrodynamic lubrication is the most important part of tribology concerning all contacts in which a lubricant fluid is interposed between two solid bodies in relative movement. This fluid may be a liquid lubricant, substantially incompressible, such as oil. In most of these mechanical systems, the inherent lubricant of mineral oil is not sufficient, chemical synthesis or dopants also called additives are mixed to the base oil to increase the performance and meet a request for increased efficiency. These additives, which are components of the engines and some industrial oils, are compounds of varied chemical structures (soluble polymers, etc.) incorporated into base oils to modify their properties. These additives are charac-

\footnotetext{
${ }^{a}$ Corresponding author:

Benyebka.Bou-Said@insa-lyon.fr
}

terized by long molecular chains which can be one million times the diameter of a water molecule and a complex rheological behavior. A number of theories have been developed to explain the peculiar behavior of the oils containing additives (polymers) [1-3]. Among them, the micro-continuum theory proposed by Vijay Kumar Stokes is the simplest theory which can describe polar effects such as the presence of couple stresses or moment of surface forces (per unit area) and body couples or moment of body forces (per unit mass or per unit volume) in addition to the body forces and surface forces per unit area or traction.

A Couple stress fluid or polar fluid is characterized by two parameters $\mu$ and $\eta_{0}$, while only one parameter is needed to characterize a Newtonian fluid that is the dynamic viscosity coefficient $\mu$. The new physical constant is due to the presence of the couple stresses in the fluid. In literature, the effect of the couple stresses on 


\section{Nomenclature}

\begin{tabular}{|c|c|}
\hline$C$ & Bearing radial clearance, $(\mathrm{m})$ \\
\hline$\tilde{C}_{\mathrm{d}}$ & Dimensionless deformation coefficient, $\left(\tilde{C}_{\mathrm{d}}=\frac{\mu_{0} \omega(R / C)^{3}}{E}\right)$ \\
\hline$C_{\mathrm{c}}$ & Coefficient of friction \\
\hline$\tilde{C}_{\mathrm{c}}$ & Dimensionless coefficient of friction, $\left(\tilde{C}_{\mathrm{c}}=\left(\frac{C}{\mu_{0} \omega L R^{3}}\right) C_{\mathrm{c}}\right)$ \\
\hline$E$ & Young's modulus of the bearing liner material, $(\mathrm{Pa})$ \\
\hline$f$ & Friction number defined by, $\left(f=\tilde{C}_{c} / \tilde{W}\right)$ \\
\hline$h$ & Fluid-film thickness, $(\mathrm{m})$ \\
\hline$\tilde{h}$ & Dimensionless fluid-film thickness, $(\tilde{h}=h / C)$ \\
\hline$L$ & Bearing length, (m) \\
\hline$\tilde{L}_{0}$ & Dimensionless compliant operator, $\left(\tilde{L}_{0}=\frac{(1+v)(1-2 v)}{(1-v)} \tilde{C}_{\mathrm{d}} \tilde{t}_{h}\right)$ \\
\hline$\ell$ & Characteristic length of additives, $\left(\ell=\sqrt{\left(\eta_{0} / \mu_{0}\right)}\right),(\mathrm{m})$ \\
\hline$\tilde{\ell}$ & Couple-stress parameter, $(\tilde{\ell}=\ell / C)$ \\
\hline$Q$ & Mean flow rate of the bearing, $\left(\mathrm{m}^{3} \cdot \mathrm{s}^{-1}\right)$ \\
\hline$\tilde{Q}$ & Dimensionless mean leakage flow of the journal bearing $\tilde{Q}=\left(\frac{L}{C \omega R^{3}}\right) Q$ \\
\hline$p$ & Fluid-film pressure, $(\mathrm{Pa})$ \\
\hline$\tilde{p}$ & Normalized film pressure, $\tilde{p}=\frac{p}{\mu_{0} \omega(R / C)^{2}}$ \\
\hline$R$ & Journal radius, $(\mathrm{m})$ \\
\hline$t_{\mathrm{h}}$ & Bearing-liner thickness, $(\mathrm{m})$ \\
\hline$\tilde{t}_{\mathrm{h}}$ & Dimensionless bearing-liner thickness, $\left(\tilde{t}_{\mathrm{h}}=t_{\mathrm{h}} / R\right)$ \\
\hline$u, v, w$ & Velocity components in circumferential, radial and axial directions, respectively, $\left(\mathrm{m} . \mathrm{s}^{-1}\right)$ \\
\hline$(\tilde{u}, \tilde{w})$ & Dimensionless fluid mean velocities, $\left(\tilde{u}=\frac{u}{\omega R}, \tilde{w}=\frac{L \tilde{w}}{\omega R^{2}}\right)$ \\
\hline$\tilde{W}$ & Dimensionless load-carrying capacity $\tilde{W}=\left(\tilde{F}_{\varepsilon}^{2}+\tilde{F}_{\phi}^{2}\right)^{1 / 2}$ \\
\hline$\tilde{F}_{\varepsilon}$ & Dimensionless load carrying-capacity component along the center line, $\tilde{F}_{\varepsilon}=\left(\frac{C^{2}}{\mu_{0} \omega R^{3} L}\right) F_{\varepsilon}$ \\
\hline$\tilde{F}_{\phi}$ & Dimensionless load component perpendicular to the center line, $\tilde{F}_{\varphi}=\left(\frac{C^{2}}{\mu_{0} \omega R^{3} L}\right) F_{\phi}$ \\
\hline$F_{\varepsilon}$ & Load carrying-capacity component along the center line, $(\mathrm{N})$ \\
\hline$F_{\phi}$ & Load component perpendicular to the center line, $(\mathrm{N})$ \\
\hline$z$ & Cartesian coordinate axis along the bearing axis, $(\mathrm{m})$ \\
\hline$\tilde{z}$ & Dimensionless axial coordinate, $\tilde{z}=z / L$ \\
\hline$\left(0_{0}, \varepsilon, \phi\right)$ & $\begin{array}{l}\text { Cartesian coordinate system centered at } 0_{0} \text { with unit vectors } \\
\varepsilon \text { and } \varphi \text { parallel and perpendicular, respectively, to the center line }\end{array}$ \\
\hline$\left(0_{0}, x, y\right)$ & Coordinate system related to the static load \\
\hline$\varepsilon$ & Static eccentricity ratio at the bearing mid-plane section, $\left(\varepsilon_{0}=e_{0} / C\right)$ \\
\hline$e$ & Static eccentricity, $\left(e=\left|0_{0} C_{0}\right|\right),(\mathrm{m})$ \\
\hline$\eta_{0}$ & Material constant responsible for the couple-stress property, (N.s) \\
\hline$\phi$ & Bearing attitude angle, (rad) \\
\hline$\phi_{0}$ & Static attitude angle, $(\mathrm{rad})$ \\
\hline$\mu$ & Atmospheric dynamic viscosity, (Pa.s) \\
\hline$\mu_{0}$ & Dynamic viscosity coefficient of the lubricant, (Pa.s) \\
\hline$\tilde{\mu}$ & Dimensionless dynamic viscosity coefficient of the lubricant, $\left(\tilde{\mu}=\mu / \mu_{0}\right)$ \\
\hline$\rho$ & Density of the lubricant, $\left(\mathrm{kg} \cdot \mathrm{m}^{-3}\right)$ \\
\hline$\tilde{\rho}$ & Dimensionless density of the lubricant, $\left(\tilde{\rho}=\rho / \rho_{0}\right)$ \\
\hline$\alpha$ & Piezoviscosity coefficient of the lubricant, $\left(\mathrm{Pa}^{-1}\right)$ \\
\hline$\tilde{\alpha}$ & Dimensionless piezoviscosity coefficient of the lubricant: $\tilde{\alpha}=\mu_{0} \omega\left(\frac{R}{C}\right)^{2} \alpha$ \\
\hline$\theta$ & Bearing angle with the origin at the maximum film thickness, (rad) \\
\hline$\theta_{c 0}$ & Static cavitation angle, (rad) \\
\hline$\omega$ & Journal angular velocity, $\left(\operatorname{rad} . \mathrm{s}^{-1}\right)$ \\
\hline
\end{tabular}


hydrodynamic journal bearings behavior is studied by defining a couple stresses parameter $\left(\ell=\left(\eta_{0} / \mu_{0}\right)^{1 / 2}\right)$, which physically represents the length of the molecular chain additives.

Influence of couple stresses parameter on linear static and dynamic or non-linear performances of journal bearings has been the subject of several theoretical and experimental studies. Over the past two decades, the main work are first those of Gupta and Sharma [4] in 1988, who theoretically studied the influence of couple stresses parameter and shaft rotational speed on the static characteristics of a hydrostatic thrust bearing. The results show that the existence of couple stresses in the lubricant film increases the load capacity of the bearing and reduce the flow rate of the lubricant fluid even for small values of the couple stresses parameter. Subsequently, in 1991, Bujurke and Naduvianamani [5], proposed a modified form of the Reynolds' equation based on the V.K. Stokes micro-continuum mechanics, in the case of a porous journal bearing. They have shown that the use of couple stress fluids or fluids containing additives in porous journal bearing substantially increases the load capacity and significantly reduces the friction number compared to the Newtonian fluid. Lin [6-11] studied the influence of the couple stresses parameter on the static and linear dynamic behavior of journal bearings. He showed that the presence of additives in the lubricant film can significantly improve the linear static and dynamic performances of the journal bearing compared to the journal bearing lubricated by Newtonian fluid. In 2001, Esharkawy and Guedouar [12] have studied the influence of couple stress parameter and elastic deformations of the bearing liner on the pressure field of a finite length journal bearing. They show firstly that the existence of couple stresses in the lubricant increases the peak pressure in the film especially for additives characterized by long molecular chains, and secondly, that the elastic deformation of the bearing liner of the journal bearing decreases the peak pressure in the film. In 2002, Naduvinamani et al. [13], are also concerned with the study of the influence of couple stress parameter and surface roughness on the static characteristics of a porous short bearing lubricated by polar fluids. The theory of Vijay Kumar Stokes and the stochastic model of Christensen are used to take account of both polar effects due to the additives and surface roughness. Numerical results show that the roughness effect of is more significant in the case of lubricated bearing by fluid containing additives. Hsiu-Lu Chiang et al. [14] in 2003 have investigated, in a theoretical study, the combined effects of couple stress parameter and surface roughness on the static performances of a finite length journal bearing operating in hydrodynamic regime. The theoretical results show that the effects of couple stresses and surface roughness are not negligible in the static analysis of the journal bearings. In 2004, Ma et al. [15] propose a theoretical study on the influence of couple stress parameter on the static characteristics of a journal bearing lubricated by fluid doped with additives, operating in hydrodynamic regime. The presence of additives in the lubricant gives a significant increase of attitude angle and pressure field in the film and a decrease of the friction coefficient of the journal bearing, especially for large values of the eccentricity ratio and couple stress parameter. In 2005, Lahmar [16] showed in an elastohydrodynamic (EHD) analysis of double-layered journal bearing lubricated with couple stress fluids that the presence of couple stresses in the lubricant for higher values of operating eccentricity led to both static peak pressure and load-carrying capacity increase with the increase of the couple stress parameter while the static attitude angle and the friction coefficient decrease, the stiffness and damping coefficients increase with couple stress parameter especially for high values of eccentricity ratio as well as the domain of stability.

In 2006, Naduvinamani and Siddangouda [17] investigated the analysis of the combined effects of the couple stress parameter and the surface roughness on the static performance of a porous thrust rectangular skid corner operating in hydrodynamic lubrication. Numerical results showed that the presence of couple stresses in the lubricant film increases the load capacity and reduces the friction coefficient of a rough porous thrust compared to a plain porous thrust. Cai-Wan Chang-Jian and Chen Chao-Kuang [18] in 2007 presented a theoretical analysis of the dynamic behavior of a flexible rotor supported by two short hydrodynamic bearings lubricated by a couple stress fluid. Numerical results show that the addition of additives in the lubricant leads to a strong reduction of vibration amplitudes and an increase of rotor-bearing system stability. In 2008, Boucherit et al. [19] present a theoretical and numerical study of the static behavior, linear and nonlinear dynamics of a journal bearing lubricated by couple stress fluid (polar fluid) considering the shaft misalignment in the journal bearing. Compared to Newtonian oils, oils with additives allow:

- A significant increase in the load-carrying capacity and static peak pressure in the lubricant especially in the case of misaligned journal bearing.

- A decrease in the power loss in the film, the attitude angle and side leakage flow for large values of static eccentricity ratio.

- The domain of stability increases when increasing the couple stress parameter especially in the case of a misaligned journal bearing even for low values of the relative eccentricity. Accordingly, the journal bearing lubricated by couple stress fluid in lightly loaded situations is more stable compared to the same journal bearing lubricated by a fluid without additives (Newtonian).

- Oils containing additives lead to a considerable decrease in the movement of the shaft center in the journal bearing and a significant increase in the minimum film thickness.

Boucherit et al. [20] have presented a comparative study of the rheological laws of lubricant fluids in hydrodynamic lubrication. Numerical results show that the presence of additives to improve the viscosity index (VI) allows to increase the hydrodynamic peak pressure in the lubricant. Boucherit and Lahmar [21] showed in a numerical study 
of the combined effects of non-Newtonian and piezoviscosity of lubricant on static performances of an infinitely long compliant journal bearing, that the fluid piezoviscosity can be neglected for the prediction of static and dynamic performances of the journal bearings severely charged. Recently, Petrone et al. [22] in 2013, have presented a study of the application of an improved Yasutomi correlation for lubricant viscosity at high pressure in a Newtonian elastohydrodynamic line contact simulation in isothermal condition. The obtained results show that the improved Yasutomi pressure-viscosity relationship derived from the free-volume model closely represents the real lubricant piezoviscous behavior for the high pressure typically encountered in elastohydrodynamic applications. We can cite also [23-25] who focus either on couple stress or piezo-viscous effects on lubricated contact performances but none on the combined effects of these phenomena.

In this study, we are interested in the study of the combined effects of the length of the molecular chains additives improving the viscosity index (polymers), viscosity-pressure variation (piezoviscosity effect) and density variation-pressure (compressibility effect) on the static behavior of a finite length plain compliant (elastic liner) journal bearing operating in isothermal condition and laminar flow. The displacement field at the fluid film bearing liner interface due to pressure forces is determined using the elastic thin liner model.

\section{Hydrodynamic lubrication by piezoviscous couple stress fluid}

\subsection{Momentum and piezoviscous couple stress fluid equations}

Based on the theory of the micro-continuum from V.K. Stokes [1], the equations of motion of a piezoviscous couple stress fluid where the body forces and couple forces are negligible, in Cartesian coordinates are:

$$
\rho \frac{\mathrm{D} \vec{V}}{\mathrm{D} t}=-\vec{\nabla} p+\vec{\nabla}(\mu \vec{\nabla} \vec{V})-\eta_{0} \vec{\nabla}^{4} \vec{V}
$$

where, $\vec{V}$ is the velocity vector of a fluid particle, $p$ is the pressure, $\rho$ and $\mu_{0}$ are respectively the density and the dynamic viscosity coefficient of the fluid and $\eta$ is a physical constant due to the presence of couple stresses in the fluid.

The continuity equation for a compressible fluid in steady state is:

$$
\frac{\partial(\rho u)}{\partial x}+\frac{\partial(\rho v)}{\partial y}+\frac{\partial(\rho w)}{\partial z}=0
$$

with, $u, v$ and $w$ the components of the velocity vector of a fluid particle.

Taking into account the assumptions of thin film lubricant [26], the equations of motion for a piezoviscous couple stress fluid (1) reduce to:

$$
\left\{\begin{array}{l}
\frac{\partial p}{\partial x}=\frac{\partial}{\partial y}\left(\mu \frac{\partial u}{\partial y}\right)-\eta_{0} \frac{\partial^{4} u}{\partial y^{4}} \\
\frac{\partial p}{\partial y}=0 \\
\frac{\partial p}{\partial z}=\frac{\partial}{\partial y}\left(\mu \frac{\partial w}{\partial y}\right)-\eta_{0} \frac{\partial^{4} w}{\partial y^{4}}
\end{array}\right.
$$

The second equation of the system (3b) shows that the pressure in the fluid does not depend on the variable $y$.

To characterize the piezoviscosity effect (viscositypressure) and the effect of compressibility (densitypressure), we use the Barus [27] and Dowson and Higginson [28] relationships in isothermal condition:

$$
\begin{aligned}
& \mu(p)=\mu \exp (\alpha p) \\
& \rho(p)=\rho_{0}\left(\frac{0.59 \times 10^{9}+1.34 \times p}{0.59 \times 10^{9}+p}\right)
\end{aligned}
$$

where $\mu_{0}$ and $\rho_{0}$ are respectively the dynamic viscosity and density of the fluid at atmospheric pressure and $\alpha$ is the piezoviscosity coefficient which depends on the lubricant oil nature, with values generally between 5 and $40 \mathrm{GPa}^{-1}[29]$ for mineral oils.

\subsection{Velocity field of a piezoviscous couple stress fluid}

The boundary conditions on the contact surfaces for the velocity (Fig. 1) are:

- For $y=0$ :

$$
\begin{array}{r}
u(x, o, z)=U_{1}=0 \quad \text { et } w(x, o, z)=W_{1}=0 \\
\left.\frac{\partial^{2} u}{\partial y^{2}}\right|_{y=0}=\left.\frac{\partial^{2} w}{\partial y^{2}}\right|_{y=0}=0
\end{array}
$$

- For $y=h$ :

$$
\begin{array}{r}
u(x, h, z)=U_{2} \quad \text { et } w(x, h, z)=W_{2}=0 \\
\left.\frac{\partial^{2} u}{\partial y^{2}}\right|_{y=h}=\left.\frac{\partial^{2} w}{\partial y^{2}}\right|_{y=h}=0 \\
v(x, o, z)=V_{1}=0 \quad \text { et } \quad v(x, h, z)=V_{2}
\end{array}
$$

$U_{2}$ and $V_{2}$ are the normal and tangential components of the velocity vector.

Equations (6) and (8) are the non-slipping conditions between the fluid and the contact surfaces and Equations (7) and (9) express the absence of couple stresses at the surfaces.

The integration of the equations of motion (3a) and $(3 \mathrm{c})$ with the boundary conditions $(6 \div 9)$ leads to 


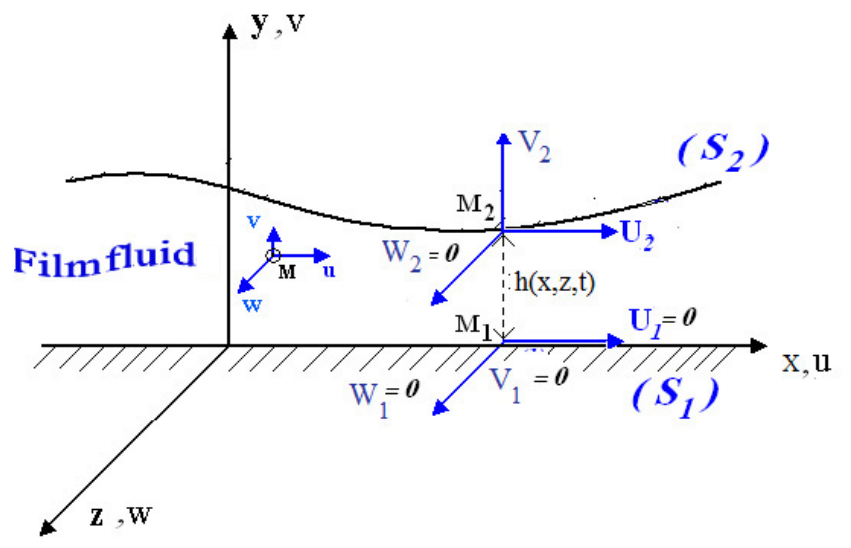

Fig. 1. System of axes.

$u$ and $w$ velocity components (Appendix):

$$
\left\{\begin{array}{l}
u=U_{2} \frac{y}{h}+\frac{\exp (-2 \alpha p)}{2 \mu_{0}} \frac{\partial p}{\partial x}\left\{\frac{y^{2}-y h}{\exp (-\alpha p)}+2 \ell^{2}\right. \\
-2 \ell^{2} \frac{\cosh [(2 y-h) /(2 \ell \exp (-0.5 \alpha p))]}{\cosh [h /(2 \ell \exp (-0.5 \alpha p))]} \\
w=\frac{\exp (-2 \alpha p)}{2 \mu_{0}} \frac{\partial p}{\partial z}\left\{\frac{y^{2}-y h}{\exp (-\alpha p)}+2 \ell^{2}\right. \\
-2 \ell^{2} \frac{\cosh [(2 y-h) /(2 \ell \exp (-0.5 \alpha p))]}{\cosh [h /(2 \ell \exp (-0.5 \alpha p))]}
\end{array}\right.
$$

where, $\ell=\left(\eta_{0} / \mu_{0}\right)^{1 / 2}$ is the couple stress parameter, which physically represents the length of the molecular chain of additives $(\ell \rightarrow 0$ in the case of a Newtonian fluid).

\subsection{Modified Reynolds equation}

After substituting Equations (11a) and (11b) in equation of continuity (2) and integrating this equation across the film thickness (Fig. 1) with boundary conditions (10), one obtains the modified Reynolds equation for an isothermal laminar flow:

$$
\begin{aligned}
\frac{\partial}{\partial x}\left(\rho G_{\mathrm{m}}(h, \ell, p) \frac{\partial p}{\partial x}\right) & +\frac{\partial}{\partial z}\left(\rho G_{\mathrm{m}}(h, \ell, p) \frac{\partial p}{\partial z}\right) \\
& =12 \mu_{0}\left(\frac{U_{2}}{2} \frac{\partial(\rho h)}{\partial x}+\rho \frac{\partial h}{\partial t}\right)
\end{aligned}
$$

where

$$
\begin{aligned}
G_{\mathrm{m}}(\ell, h, p) & =h^{3} \exp (-\alpha p)-12 h \ell^{2} \exp (-2 \alpha p) \\
& +24 \ell^{3} \exp (-2.5 \alpha p) \tanh (h /(2 \ell \exp (-0.5 \alpha p))
\end{aligned}
$$

Equation (12) is the basic equation of hydrodynamic lubrication for a barotropic and piezoviscous couple stress fluid written for a two-dimensional flow (2D).

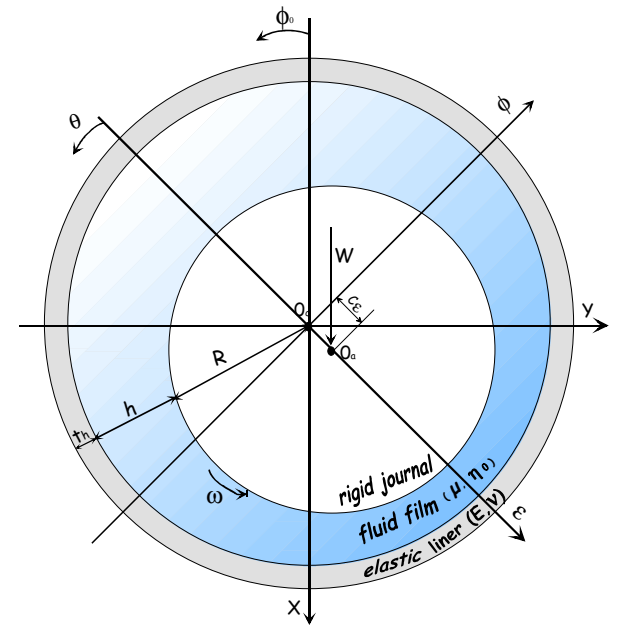

Fig. 2. Cross-section of a plain compliant journal bearing.

For a finite length smooth journal bearing (Fig. 2), the modified Reynolds Equation (12), in steady-state becomes:

$$
\begin{array}{r}
\frac{\partial}{R^{2} \partial \theta}\left(\rho G_{\mathrm{m}}(h, \ell, p) \frac{\partial p}{\partial \theta}\right)+\frac{\partial}{\partial z}\left(\rho G_{\mathrm{m}}(h, \ell, p) \frac{\partial p}{\partial z}\right) \\
=6 \mu_{0} \omega \frac{\partial}{\partial \theta}(\rho h)
\end{array}
$$

where, $U_{2}=\omega \times R$ et $\theta=x / R$.

$\omega$ and $R$ are the angular velocity and the journal radius, respectively and $h$ is the film thickness.

\subsection{Lubricant film thickness}

In the case of an aligned smooth rigid journal bearing, the geometric equation which defines at each point the lubricant film thickness is given by:

$$
h(\theta, z)=C(1+\varepsilon \cos \theta)
$$

where $C$ is the radial clearance and $\varepsilon$ is the static eccentricity ratio of the journal bearing.

For numerical treatment, it is recommended to rewrite all the equations of hydrodynamic lubrication in dimensionless variables. In the case of a journal bearing, we have:

$$
\begin{aligned}
\tilde{h}=\frac{h}{C}, \quad \tilde{z}=\frac{z}{L}, \quad \tilde{\mu}=\frac{\mu}{\mu_{0}}, \quad \tilde{\rho}=\frac{\rho}{\rho_{0}}, \quad \tilde{\ell}=\frac{\ell}{C}, \\
\text { and } \quad \tilde{p}=\frac{p}{\mu_{0} \omega(R / C)^{2}}
\end{aligned}
$$

with, $L$ the bearing length.

Thus, the dimensionless modified Reynolds Equation (13) can be written as:

$$
\begin{aligned}
\frac{\partial}{\partial \theta}\left(\tilde{\rho} \tilde{G}_{\mathrm{m}}(\tilde{h}, \tilde{\ell}, \tilde{p}) \frac{\partial \tilde{p}}{\partial \theta}\right)+\left(\frac{R}{L}\right)^{2} \\
\quad \times \frac{\partial}{\partial \tilde{z}}\left(\tilde{\rho} \tilde{G}_{\mathrm{m}}(\tilde{h}, \tilde{\ell}, \tilde{p}) \frac{\partial \tilde{p}}{\partial \tilde{z}}\right)=6 \frac{\partial}{\partial \theta}(\tilde{\rho} \tilde{h})
\end{aligned}
$$


where,

$$
\begin{aligned}
\tilde{G}_{\mathrm{m}}(\tilde{\ell}, \tilde{h}, \tilde{p}) & =\tilde{h}^{3} \exp (-\tilde{\alpha} \tilde{p})-12 \tilde{h} \tilde{\ell}^{2} \exp (-2 \tilde{\alpha} \tilde{p}) \\
& +24 \tilde{\ell}^{3} \exp (-2.5 \tilde{\alpha} \tilde{p}) \tanh (\tilde{h} /(2 \tilde{\ell} \exp (-0.5 \tilde{\alpha} \tilde{p})))
\end{aligned}
$$

Barus and Dowson (4) and Higginson (5) relationships become:

$$
\begin{aligned}
& \tilde{\mu}=\exp (\tilde{\alpha} \tilde{p}) \\
& \tilde{\rho}=\left(\frac{0.59 \times 10^{9}+1.34 \tilde{p}}{0.59 \times 10^{9}+\tilde{p}}\right)
\end{aligned}
$$

where $\tilde{\alpha}$ is the dimensionless fluid piezoviscosity coefficient: $\tilde{\alpha}=\mu_{0} \omega\left(\frac{R}{C}\right)^{2} \alpha$.

The dimensionless film thickness is:

$$
\tilde{h}=1+\varepsilon \cos \theta
$$

Taking into account the elastic deformation of the bearing liner led to the modification of the geometrical film Equation (18). For an aligned compliant journal bearing, and if the thin elastic liner model is used, the expression of dimensionless static film thickness (Eq. (18)) becomes:

$$
\tilde{h}=1+\varepsilon \cos \theta+\tilde{L}_{0} \tilde{p}
$$

where, $\tilde{L}_{0}$ is the dimensionless compliance operator given by: $\tilde{L}_{0}=\frac{(1+v)(1-2 v)}{(1-v)} \tilde{C}_{d} \tilde{t}_{h}, \tilde{p}$ is the static hydrodynamic pressure, $\tilde{t}_{h}=t_{h} / R$ is the relative thickness of the bearing-liner, and $\tilde{C}_{d}=\frac{\mu_{0} \omega(R / C)^{3}}{E}$ is the dimensionless deformation coefficient inversely proportional to the elasticity modulus, $E$ and $v$ are the Young's modulus and Poisson's ratio of bearing-liner material, respectively.

The (three-dimensional [3-D]) thin elastic liner model (the deformations at the fluid liner interface are assumed to be proportional to the static hydrodynamic pressure) is used to account for the effects of elastic deformation of the bearing liner. Compared to the finite element method (FEM), the main advantage of this elasticity model is its low consuming computational time, since practically a very short CPU time is needed to compute the elastic deformations [19].

\subsection{Pressure boundary conditions}

The pressure field in the film must satisfy the modified Reynolds equation and the following boundary conditions:

$$
\begin{aligned}
& \tilde{p}=0 \quad \text { at } \quad \tilde{z}= \pm \frac{1}{2} \\
& \tilde{p}=0 \quad \text { at } \quad \theta=0 \quad \text { where } \theta=2 \pi \\
& \tilde{p}=\frac{\partial \tilde{p}}{\partial \theta}=0 \quad \text { at } \quad \theta=\theta_{\mathrm{Ca}}
\end{aligned}
$$

Condition (20) implies that the pressure at the bearing ends equals atmospheric pressure, while the conditions (22) are the Swift-Stieber boundary conditions known as the Reynolds conditions. $\theta_{\text {ca }}$ is the film rupture angle, so-called the cavitation angle measured from the maximum film thickness at which cavitation starts.

\section{Static characteristics of journal bearing}

From the numerical resolution of the modified Reynolds Equation (16), we obtain the pressure field in the film. From the pressure, we can calculate the static characteristics, such as the load-carrying capacity, the static attitude angle, the coefficient of friction, the mean velocity fields of the flow as well as the mean leakage flow at each end of the journal bearing.

\subsection{Load-carrying capacity and attitude angle}

In the intermediate plan $\left(O_{\mathrm{c}}, \vec{\varepsilon}, \vec{\phi}, \vec{z}\right)$, (Fig. 2), the load-carrying capacity and the attitude angle are given as:

$$
\begin{aligned}
& \tilde{W}=\left(\tilde{F}_{\varepsilon}^{2}+\tilde{F}_{\phi}^{2}\right)^{1 / 2} \\
& \phi_{0}=\tan ^{-1}\left(-\frac{\tilde{F}_{\phi}}{\tilde{F}_{\varepsilon}}\right)
\end{aligned}
$$

where, $\tilde{F}_{\varepsilon}=\left(\frac{C^{2}}{\mu_{0} \omega R^{3} L}\right) F_{\varepsilon}=\int_{0}^{2 \pi} \int_{-1 / 2}^{1 / 2} \tilde{p} \sin \theta \mathrm{d} \tilde{z} \mathrm{~d} \theta$ and $\tilde{F}_{\phi}=\left(\frac{C^{2}}{\mu_{0} \omega R^{3} L}\right) F_{\phi}=\int_{0}^{2 \pi} \int_{-1 / 2}^{1 / 2} \tilde{p} \sin \theta \mathrm{d} \tilde{z} \mathrm{~d} \theta$.

\subsection{Coefficient of friction on the housing}

The coefficient of friction is obtained by integration of the shear stresses at the bearing surface, in dimensionless variables:

$$
\begin{aligned}
\tilde{C}_{c} & =\left(\frac{C}{\mu_{0} \omega L R^{3}}\right) C_{c} \\
& =\int_{-1 / 2}^{1 / 2} \int_{0}^{2 \pi}\left(\frac{\exp (\tilde{\alpha} \tilde{p})}{\tilde{h}}-\frac{1}{2} \frac{\partial \tilde{p}}{\partial \theta}\{\tilde{h}-2 \tilde{\ell} \exp (-0.5 \tilde{\alpha} \tilde{p})\right. \\
& \times \tanh [\tilde{h} /(2 \tilde{\ell} \exp (-0.5 \tilde{\alpha} \tilde{p}))]\}) \mathrm{d} \theta \mathrm{d} \tilde{z}
\end{aligned}
$$

The friction number is defined as: $f=\frac{\tilde{C}_{c}}{\tilde{W}}$.

\subsection{Mean velocities of the flow and mean leakage flow}

The mean velocities of the fluid flow are provided by the following expression:

$$
\left\{\begin{array}{l}
u=\frac{1}{h} \int_{0}^{h} u \mathrm{~d} y=\frac{\omega R}{2}-\frac{1}{h} \frac{G_{m}(h, \ell, p)}{12 \mu_{0}} \frac{\partial p}{R \partial \theta} \\
w=\frac{1}{h} \int_{0}^{h} w \mathrm{~d} y=-\frac{1}{h} \frac{G_{\mathrm{m}}(h, \ell, p)}{12 \mu_{0}} \frac{\partial p}{\partial z}
\end{array}\right.
$$

The dimensionless mean velocities of the flow become:

$$
\left\{\begin{array}{l}
\tilde{u}=\frac{u_{\mathrm{m}}}{\omega R}=\frac{1}{2}-\frac{\tilde{G}_{\mathrm{m}}(\tilde{h}, \tilde{\ell}, \tilde{p})}{12 \tilde{h}} \frac{\partial \tilde{p}}{\partial \theta} \\
\tilde{w}=\frac{L w_{\mathrm{m}}}{\omega R^{2}}=-\frac{\tilde{G}_{\mathrm{m}}(\tilde{h}, \tilde{\ell}, \tilde{p})}{12 \tilde{h}} \frac{\partial \tilde{p}}{\partial \tilde{z}}
\end{array}\right.
$$


A. Mouassa et al.: Mechanics \& Industry 16, 608 (2015)

Table 1. Parameter values for numerical calculations.

\begin{tabular}{lc}
\hline Geometric characteristics & \\
\hline Shaft radius, $R$ & $25 \times 10^{-3} \mathrm{~m}$ \\
Bearing length, $L$ & $50 \times 10^{-3} \mathrm{~m}$ \\
Radial clearance, $C$ & $7 \times 10^{-5} \mathrm{~m}$ \\
\hline Operating Conditions & $100 \times \pi$ rad.s ${ }^{-1}$ \\
\hline Shaft rotational speed, $\omega$ & \\
\hline Lubricant property parameters & $0.03 \mathrm{~Pa} . \mathrm{s}$ \\
\hline Atmospheric dynamic viscosity, $\mu_{0}$ & $870 \mathrm{~kg} . \mathrm{m}^{-3}$ \\
Atmospheric density, $\rho_{0}$ & 0,10 et $30 \times 10^{-9} \mathrm{~Pa}^{-1}$ \\
Piezoviscosity coefficient, $\alpha$ & \\
\hline Bearing liner characteristics & $126 \mathrm{GPa}$ \\
\hline Young modulus, E & 0.3 \\
Poisson's coefficient, $\nu$ & $0.005 \mathrm{~m}$ \\
Thickness of the thin elastic liner, th &
\end{tabular}

The mean flow at the bearing end is expressed by:

$$
Q=\left.\int_{0}^{2 \pi} \int_{0}^{h} w\right|_{z=L / 2} R \mathrm{~d} y \mathrm{~d} \theta
$$

In dimensionless variable becomes:

$$
\tilde{Q}=\left(\frac{L}{C \omega R^{3}}\right) Q=\left|\int_{0}^{2 \pi}\left[-\left.\frac{\tilde{G}_{\mathrm{m}}(\tilde{h}, \tilde{\ell}, \tilde{p})}{6} \frac{\partial \tilde{p}}{\partial \tilde{z}}\right|_{\tilde{z}=1 / 2}\right] \mathrm{d} \theta\right|
$$

\section{Method of resolution}

In the case of a journal bearing lubricated by barotropic and piezoviscous couple stress fluid, the modified Reynolds equation is discretized using the finite differences method. The obtained nonlinear algebraic equations system are solved by iterative numerical procedure. A flow chart is given in the following (Fig. 3):

\section{Results and discussion}

For validation we study the influence of fluid rheology on the static behavior of the journal bearing and compare the obtained results with those available in the literature.

Figure 4 represents the circumferential variations of the dimensionless pressure in the middle section of a rigid finite journal bearing $(R / L=0.5)$ for various values of couple stress parameter $(\tilde{\ell}=0.0, \tilde{\ell}=0.2$ et $\tilde{\ell}=0.4)$ and a static eccentricity ratio $\varepsilon_{0}=0.9$. As shown in the figure, the results obtained are in good agreement with those published by Kabouya et al. [30].

\subsection{Parametric study}

In this parametric study, we will investigate the influence of the couple stress parameter $(\tilde{\ell})$, viscosity-pressure variation (piezoviscosity effect) and density-pressure variation (compressibility effect) on the field pressure, loadcarrying capacity, attitude angle friction number and side leakage flow of a finite length plain journal bearing $(R / L=0.5)$ lubricated with a barotropic and piezoviscous couple stress fluid which geometrical characteristics, operating conditions and physical properties of the lubricant are given in Table 1.

\subsubsection{Influence couple stress parameter, piezoviscosity and compressibility of the lubricant on the pressure field}

We studied the combined effects of the couple stress parameter, the piezoviscosity and the compressibility of the lubricant on the maximum field pressure in the film. The calculations were made for a static eccentricity ratio $\varepsilon=0.9$, for various values of couple stress $(\tilde{\ell}=0.0$ (newtonian fluid), 0.2 et 0.3 ) and four types of polar lubricant fluid:

- incompressible and isoviscous fluid: $\tilde{\rho}=$ cte et $\tilde{\mu}=$ cte;

- compressible and isoviscous fluid: $\tilde{\rho}=\tilde{\rho}(p)$ et $\tilde{\mu}=$ cte;

- incompressible and piezoviscous fluid: $\tilde{\rho}=$ cte et $\tilde{\mu}(\tilde{p})=\exp (\tilde{\alpha} \tilde{p})$

- compressible and piezoviscous fluid: $\tilde{\rho}=\tilde{\rho}(\tilde{p})$ et $\tilde{\mu}(\tilde{p})=\exp (\tilde{\alpha} \tilde{p})$.

Figure 5 shows the circumferential variations of hydrodynamic pressure field in the middle section of the journal bearing for different conditions. There is a significant increase of the peak pressure in a journal bearing lubricated by fluid with additives (polar fluid) compared to the journal bearing lubricated with Newtonian fluid (Figs. 5a-5f). This increase becomes very important in the order of $97 \%$ with the increase of couple stress parameter $(\tilde{\ell}=0.3)$ and piezoviscosity coefficient $\left(\alpha=30 \mathrm{GPa}^{-1}\right)$ (Figs. 5a, 5d and $5 \mathrm{f}$ ). For the same values of couple stress parameter the lubricant piezoviscosity leads to a fairly significant increase of peak pressure in the film (Figs. 5a-5d, 5b-5f and $6 \mathrm{a}-6 \mathrm{c})$. It is concluded that the piezoviscosity of the fluid cannot be overlooked for the prediction of static performances especially if the bearing is severely loaded. In 
A. Mouassa et al.: Mechanics \& Industry 16, 608 (2015)

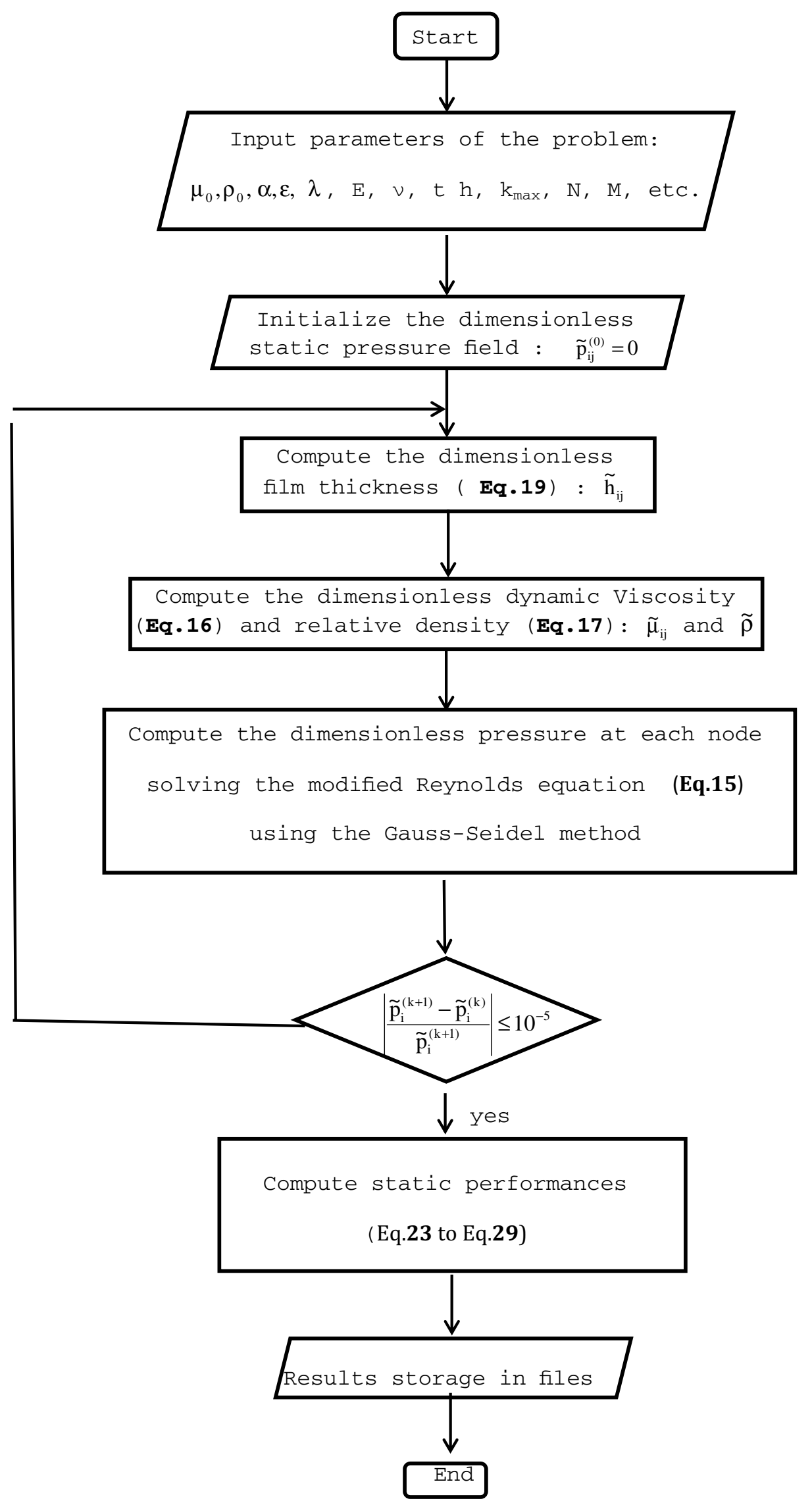

Fig. 3. Flow chart. 


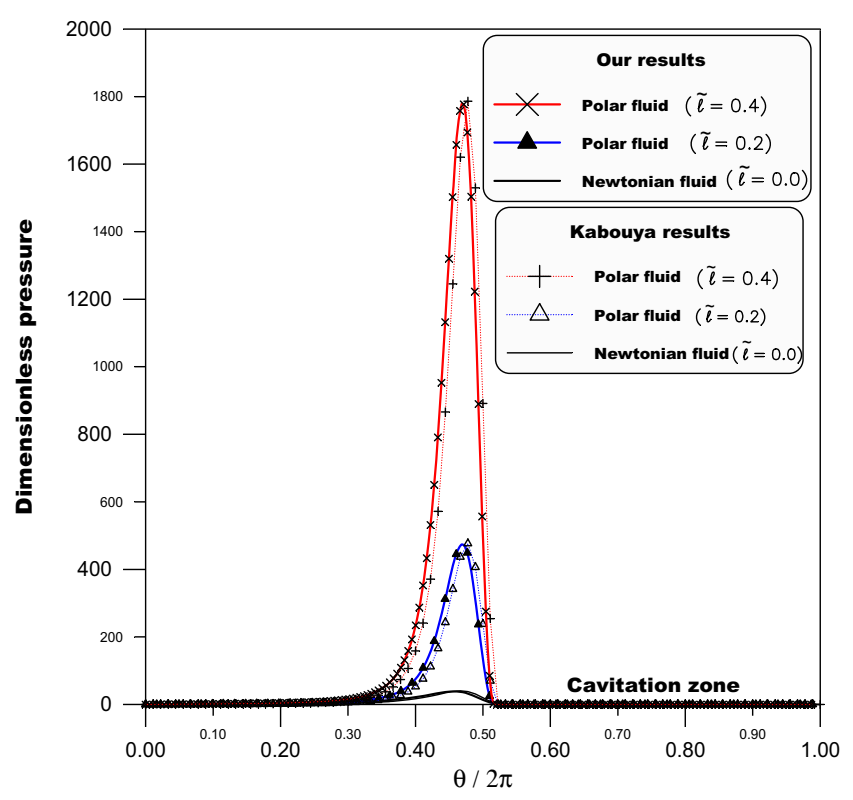

Fig. 4. Circumferential variations of dimensionless pressure rescaled in the midsection of journal bearing for various values of couple stress.

this case the effect of piezoviscosity becomes significant because of high pressure values especially at high eccentricity running conditions.

The compressibility of the lubricant fluid has no significant effect on the pressure field (Figs. 5a-5b, 5c-5e and $5 \mathrm{~d}-5 \mathrm{f}$ and $6 \mathrm{a}-6 \mathrm{~b})$ in this case because of the nature of lubricant.

\subsubsection{Influence of couple stress parameter and piezoviscosity of the lubricant on static performances}

Static performances of finite compliant journal bearing were obtained for static eccentricity ratio $\varepsilon$ ranging from 0.05 to 0.90 , and different values of dimensionless couple stress parameter $(\tilde{\ell}=0.0,0.1$ and 0.3$)$ and piezoviscosity coefficient $\left(\alpha=10 \mathrm{GPa}^{-1}\right.$ and $\left.\alpha=30 \mathrm{GPa}^{-1}\right)$.

Figure 6 presents the load-carrying capacity of the journal bearing for different static eccentricity ratios and couple stress parameters $(\tilde{\ell})$. The figure shows a significant increase of the load-carrying capacity with the couple stress parameter increase especially for large values of static eccentricity ratio (heavily loaded bearing). This increase is very important for couple stress parameter $\tilde{\ell}=0.3$ and a piezoviscosity coefficient $\alpha=30 \mathrm{GPa}^{-1}$ for a heavily loaded journal bearing $(\varepsilon=0.9)$. The parameter $\tilde{\ell}$ was chosen to vary in the range of 0 . to 0.3 as usually found in literature. These values are theoretical and it is to notice that from our best knowledge there is no experimental evidences to validate these values. 0.3 value has been taken to amplify the couple stress effects and can be considered as an extreme value.

It can be concluded that the presence of large molecular chains additives in lubricant oils and piezoviscosity of fluid improves the load capacity of journal bearings compared to journal bearing lubricated with pure mineral oil.

The variations of friction number and average flow rate according to the static relative eccentricity for different values of the couple stress parameter of a journal bearing lubricated by piezoviscous polar fluid are shown in Figures 8 and 9 .

As shown in these figures the increase of couple stress parameter leads to a decrease in friction number; this decrease is more significant (in the order of $68 \%$ ) in the case of high value of the couple stress parameter $(\tilde{\ell}=0.3)$ and heavily loaded bearing $(\varepsilon=0.9)$. The average flow rate decreases especially for large values of static eccentricity ratio.

Additives in oils can significantly reduce the energy losses by friction in the lubricant particularly in the case of heavily loaded journal bearings. This comment must be confirmed with a thermohydrodynamic investigation.

Piezoviscosity has no effect because of large contact conditions which leads to pressure values not sufficient to "activate" this property.

The flow rate is not really affected by couple stress effect. This result is consistent with those found in literature on non-Newtonian studies [20].

\section{Conclusion}

Our theoretical and numerical study aims essentially to highlight the influence of the combined effects of couple stress parameter, viscosity-pressure dependency (piezoviscosity effects) and density-pressure variation (compressibility effect) on the static performances of a finite length compliant journal bearing operating in isothermal and laminar conditions. We based our approach on the Micro-continuum V.K. Stokes theory to describe the movement of polar fluids. Barus and Dowson and Higginson laws are held to account for both viscositypressure variation and density-pressure variation effects. The problem is solved numerically by iterative process.

The results show that the presence in the lubricant of additives improving the viscosity index (VI) and piezoviscosity effects has a significant influence on the static performances of journal bearings especially for large values of couple stress parameter. Compared to Newtonian oils, oils with additives allow:

- A significant increase in peak pressure in the film and hydrodynamic load-carrying capacity especially for large values of couple stress parameter and static eccentricity ratio.

- A decrease of friction number in the film and average flow rate for large values of static relative eccentricity. Accordingly, couple stress effects significantly reduce the energy losses by friction in the lubricant in the above cases where the bearing is heavily loaded.

For the same values of couple stress parameter the piezoviscosity leads to an increase of peak pressure in the film 
A. Mouassa et al.: Mechanics \& Industry 16, 608 (2015)

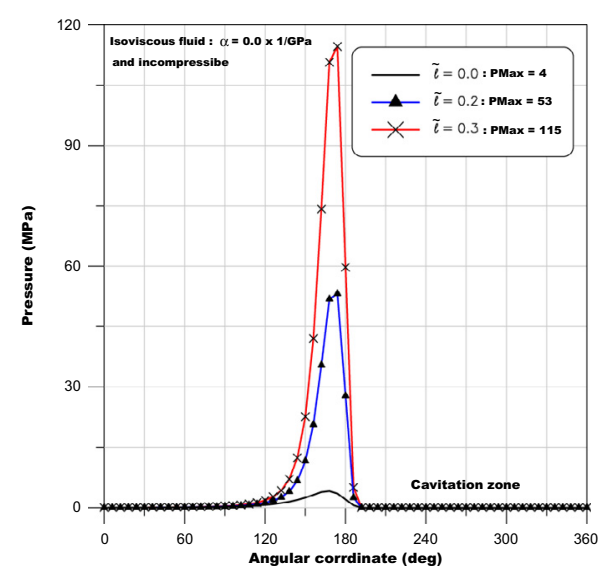

(a)

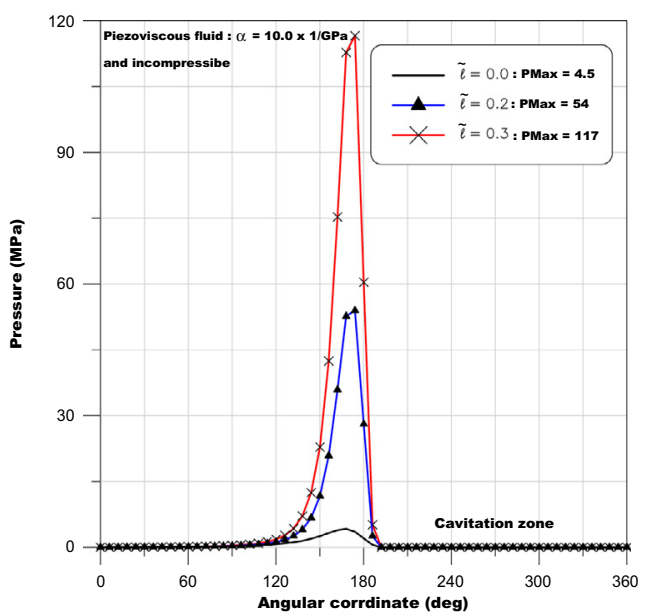

(c)

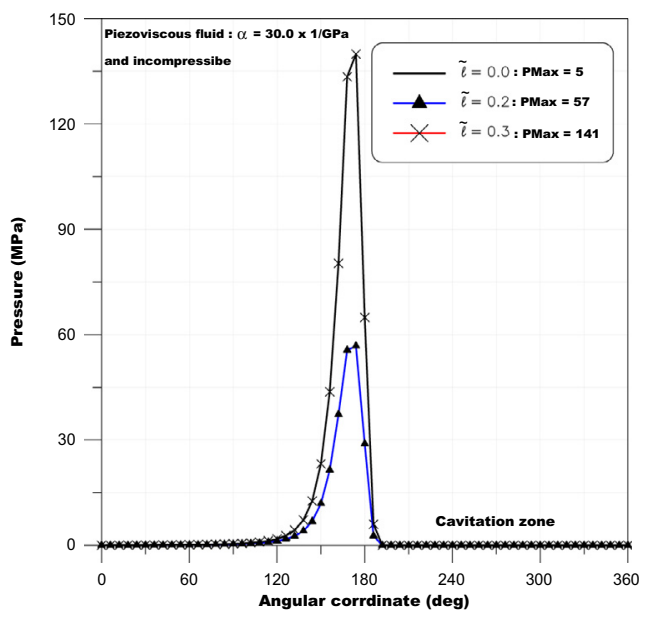

(e)

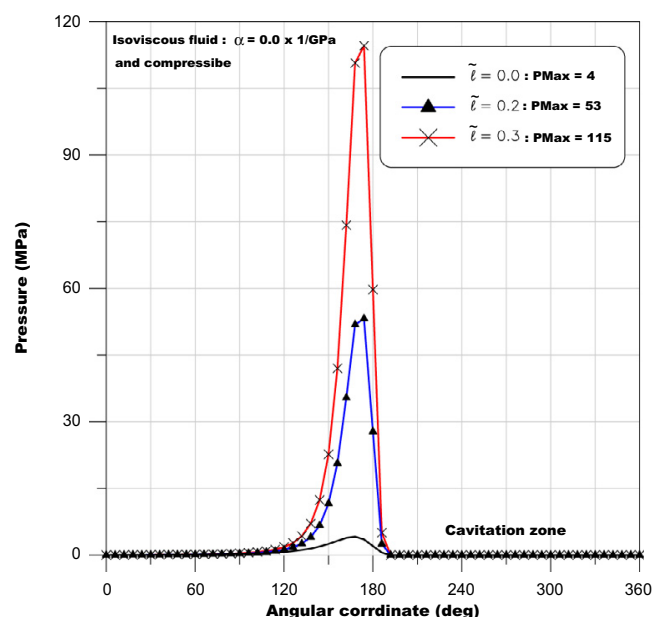

(b)

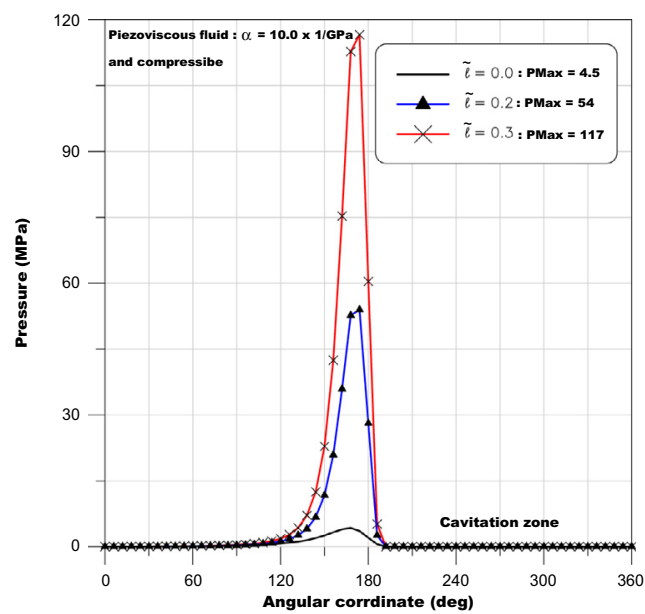

(d)

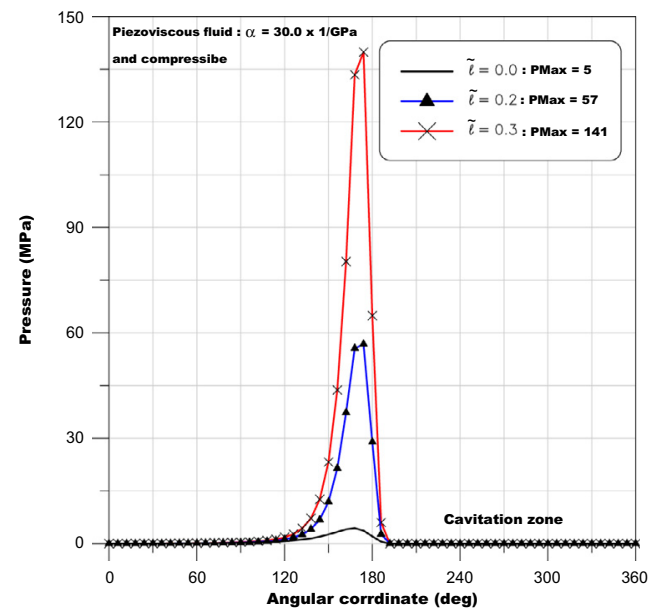

(f)

Fig. 5. Circumferential variations of pressure field in the midsection of journal bearing for different fluids $(\varepsilon=0.9)$. (a) incompressible isoviscous fluid, (b) compressible isoviscous fluid, (c) incompressible piesoviscous fluid $\left(\alpha=10 \mathrm{GPa}^{-1}\right)$, (d) compressible piesoviscous fluid $\left(\alpha=10 \mathrm{GPa}^{-1}\right)$, (e) incompressible piesoviscous fluid $\left(\alpha=30 \mathrm{GPa}^{-1}\right)$, (f) compressible piesoviscous fluid $\left(\alpha=3 \mathrm{GPa}^{-1}\right)$. 


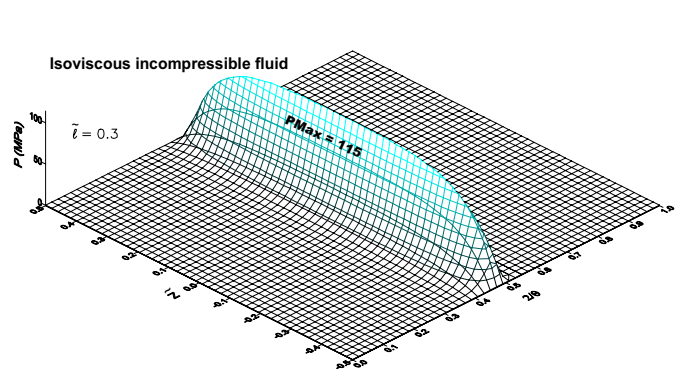

(a)

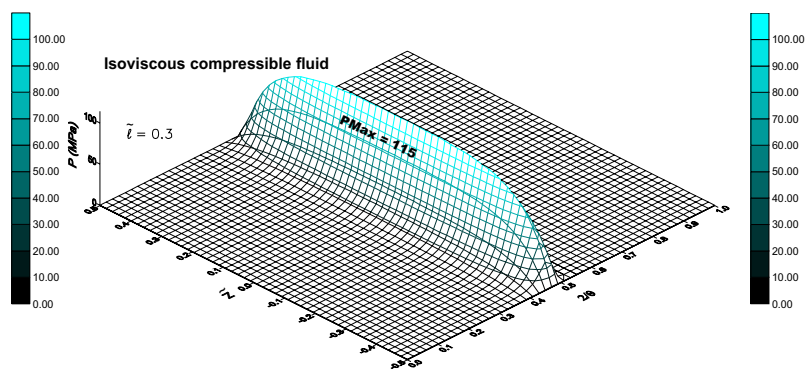

(b)

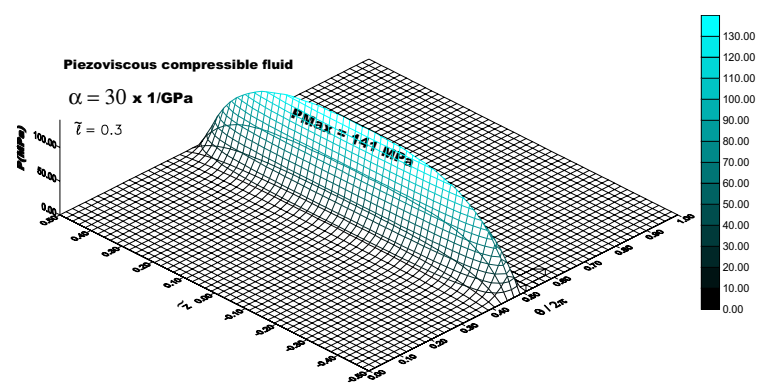

(c)

Fig. 6. 3D static pressure field for different fluids $(\varepsilon=0.9)$. (a) Incompressible isoviscous fluid, (b) compressible isoviscous fluid, (c) compressible piezoviscous fluid.

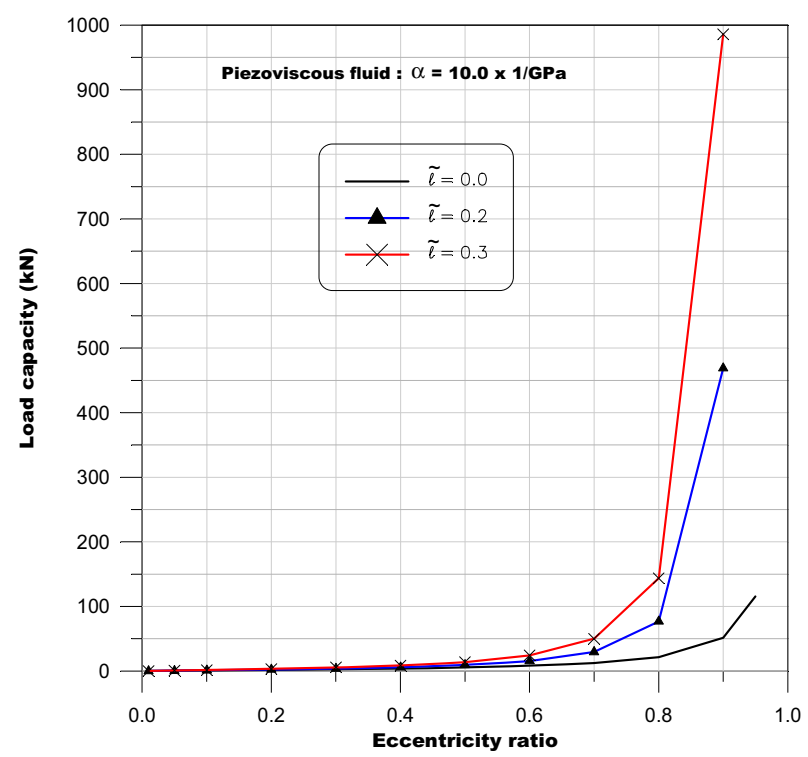

(a)

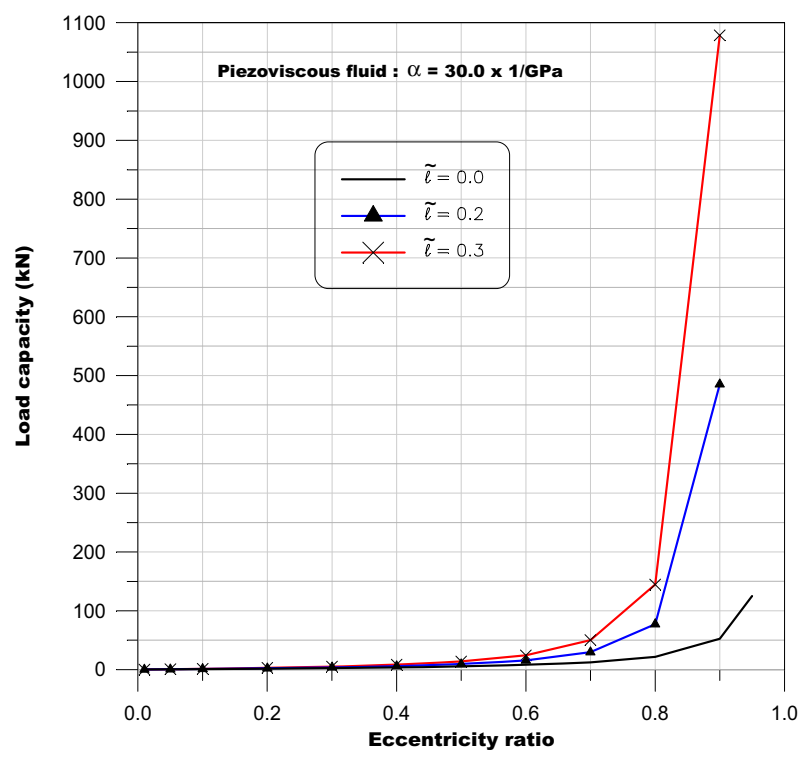

(b)

Fig. 7. Variation of carrying capacity versus static eccentricity ratio for various values of couple stress and relative piezoviscosity coefficient of lubricant. (a) Piezoviscous fluid $\left(\alpha=10 \mathrm{GPa}^{-1}\right)$, (b) piezoviscous fluid $\left(\alpha=30 \mathrm{GPa}^{-1}\right)$.

and hydrodynamic carrying capacity of the journal bearing especially in the case of heavily loaded bearing.

It can be concluded that the piezoviscosity cannot be overlooked for the prediction of static characteristics of severely contaminated fluids levels where the effects of piezoviscosity become important.

The compressibility of the fluid does not have significant effects on the pressure field in the lubricant.

\section{Appendix: Expression of flow velocity field for a piezoviscous couple stress fluid}

We suppose that: $h_{1}=0$ and $h_{2}=h(x, z, t)$. Definition of the assumptions and the boundary conditions on speeds are given hereafter:

- the fluid adheres perfectly to the surfaces; 
A. Mouassa et al.: Mechanics \& Industry 16, 608 (2015)

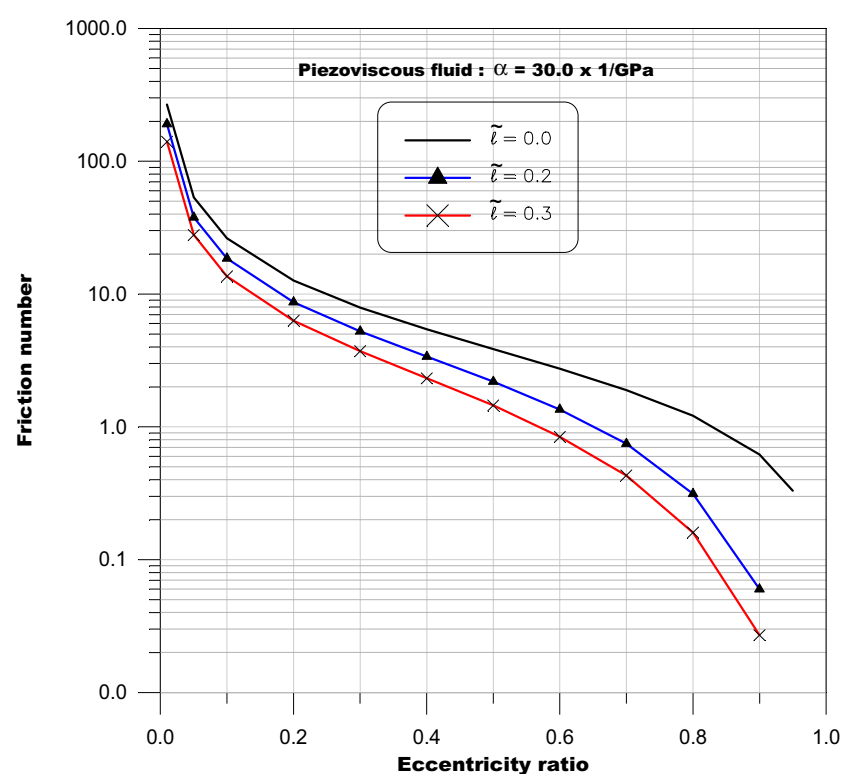

Fig. 8. Variation of friction number versus static eccentricity ratio for various values of couple stress parameter.

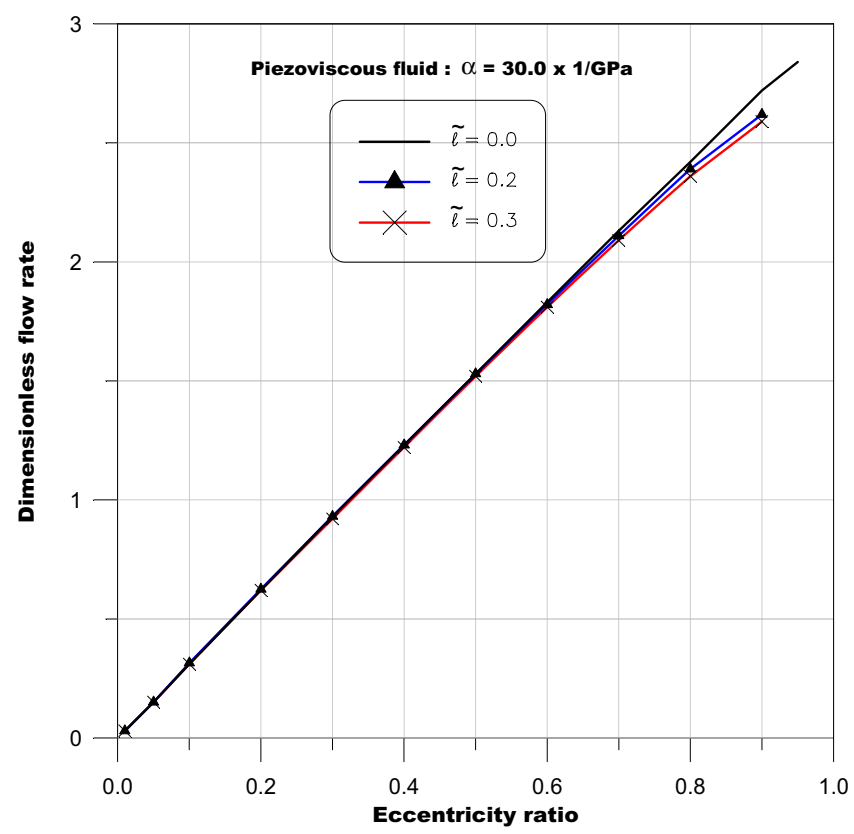

Fig. 9. Variation of dimensionless flow rate versus static eccentricity ratio for various values of couple stress parameter.

- it is supposed that the inferior surface $\left(\mathrm{S}_{1}\right)$ is plane and merged with the plan $\left(x_{1}, O, x_{3}\right)$;

- speed in all points of surface $\left(\mathrm{S}_{1}\right)$ is tangent on this surface.

For a two-dimensional flow (2D) in the thin film theory according to system of axes given in Figure 1 equations of motion (equations of Navier) (1) of a piezoviscous couple stress fluid $(\mu=f(p))$ in isothermal condition
$(\mu=f(T)=$ cts and $\eta=f(T)=$ cts $)$ are written as:

$$
\left\{\begin{array}{l}
\frac{\partial p}{\partial x}=\frac{\partial}{\partial y}\left(\mu \frac{\partial u}{\partial y}\right)-\eta_{0} \frac{\partial^{4} u}{\partial y^{4}} \\
\frac{\partial p}{\partial y}=0 \\
\frac{\partial p}{\partial z}=\frac{\partial}{\partial y}\left(\mu \frac{\partial w}{\partial y}\right)-\eta_{0} \frac{\partial^{4} w}{\partial y^{4}}
\end{array}\right.
$$

To characterize the piezoviscosity effect (viscositypressure) and the effect of compressibility (densitypressure), we use the relations of Barus (4) and Dowson and Higginson (5):

$$
\left\{\begin{array}{l}
\mu(p)=\mu_{0} \mathrm{e}^{\alpha p} \\
\frac{\rho}{\rho_{0}}=1+\frac{0.58 \times 10^{-9} p}{1+1.68 \times 10^{-9} p}
\end{array}\right.
$$

The pressure in film is not dependent of the axis $y,(p=$ $p(x, z))$, i.e.: $\frac{\partial p}{\partial y}=0$, so $\frac{\partial \mu}{\partial y}=0$.

In this case the system of Equations (A.1) and (A.3) becomes:

$$
\begin{aligned}
& \frac{\partial p}{\partial x}=\mu\left(\frac{\partial^{2} u}{\partial y^{2}}\right)-\eta_{0} \frac{\partial^{4} u}{\partial y^{4}} \\
& \frac{\partial p}{\partial z}=\mu\left(\frac{\partial^{2} w}{\partial y^{2}}\right)-\eta_{0} \frac{\partial^{4} w}{\partial y^{4}}
\end{aligned}
$$

To determine the first component of vector velocity we can write Equation (A.5a) in the following form:

$$
\eta_{0} \frac{\partial^{4} u}{\partial y^{4}}-\mu\left(\frac{\partial^{2} u}{\partial y^{2}}\right)=-\frac{\partial p}{\partial x}
$$

It is a linear equation of order four with constant coefficients, its general solution $(\mathbf{u})$ is the sum of the homogeneous solution $\left(\mathbf{u}_{\mathbf{h}}\right)$ plus the particular solution $\left(\mathbf{u}_{\mathbf{p}}\right)$.

\section{(a) Homogeneous solution}

The homogeneous solution (A.6) is found as:

$$
u_{\mathrm{h}}(y)=A_{0}+A_{1} y+C_{1} \mathrm{e}^{\sqrt{\mathrm{e}^{\alpha p}} y / \ell}+C_{2} \mathrm{e}^{-\sqrt{\mathrm{e}^{\alpha p}} y / \ell}
$$

with, $\ell=\sqrt{\frac{\eta_{0}}{\mu_{0}}}$ and $A, A_{1}, C_{0}$ and $C_{1}$ belong to $\mathfrak{R}$.

\section{(b) Particular solution}

We suppose, a polynomial solution of order two:

$$
u_{p}=a y^{2}+b y+c
$$

therefore: $\frac{\partial u_{p}}{\partial y}=2 a y+b, \frac{\partial u_{p}}{\partial y}=2 a$ and $\frac{\partial u_{p}}{\partial y}=0$.

While replacing, $\mathbf{u}$ by $\mathbf{u}_{p}$ in Equation (A.6), we obtain: $\eta(0)-\mu(2 a)=-\frac{\partial p}{\partial x}$, so, $a=\frac{1}{2 \mu_{0} \mathrm{e}^{\alpha p}} \frac{\partial p}{\partial x}$, with, $\mu=\mu_{0} \mathrm{e}^{\alpha p}$. 
There are no condition for coefficients $B$ and $C$, we choose to take $B=C=0$, and we replace these constants in the polynomial (A.8), we find: $u_{p}=\frac{1}{2 \mu_{0} \mathrm{e}^{\alpha p}} \frac{\partial p}{\partial x} y^{2}$.

At the end the general solution of Equation (A.6) is the sum of the homogeneous solution $\left(\mathbf{u}_{h}\right)$ more the particular solution $\left(\mathbf{u}_{p}\right)$ :

$$
\begin{aligned}
u=u_{\mathrm{h}}+u_{\mathrm{p}}=A_{0}+A_{1} y+\frac{1}{2 \mu_{0} \mathrm{e}^{\alpha p}} & \frac{\partial p}{\partial x} y^{2}+C_{1} \mathrm{e}^{\sqrt{\mathrm{e}^{\alpha p}} y / \ell} \\
& +C_{2} \mathrm{e}^{-\sqrt{\mathrm{e}^{\alpha p}} y / \ell}
\end{aligned}
$$

Therefore, the general solutions for rates of flow of a piezoviscous polar fluid particle are:

$$
\begin{aligned}
u(y) & =A_{0}+A_{1} y+\frac{1}{2 \mu_{0} \mathrm{e}^{\alpha p}} \frac{\partial p}{\partial x} y^{2}+B_{1} \operatorname{ch}\left(\sqrt{\mathrm{e}^{\alpha p}} y / \ell\right) \\
& +B_{2} \operatorname{sh}\left(\sqrt{\mathrm{e}^{\alpha p}} y / \ell\right) \\
w(y) & =A_{0}^{\prime}+A^{\prime}{ }_{1} y+\frac{1}{2 \mu_{0} \mathrm{e}^{\alpha p}} \frac{\partial p}{\partial z} y^{2}+B^{\prime}{ }_{1} \operatorname{ch}\left(\sqrt{\mathrm{e}^{\alpha p}} y / \ell\right) \\
& +B^{\prime}{ }_{2} \operatorname{sh}\left(\sqrt{\mathrm{e}^{\alpha p}} y / \ell\right)
\end{aligned}
$$

with $A, A_{1}, A^{\prime}, A_{1}^{\prime}, B_{0}, B_{1}, B_{0}^{\prime}$ and $B_{1}^{\prime}$ constants in $\mathfrak{R}$. And, $B_{1}=C_{1}+C_{2}$ and $B_{2}=C_{1}-C_{2}$.

where:

$$
\begin{aligned}
C_{1} \mathrm{e}^{\sqrt{\mathrm{e}^{\alpha p}} y / \ell}+C_{2} \mathrm{e}^{-\sqrt{\mathrm{e}^{\alpha p}} y / \ell}= & B_{1}\left(\frac{\mathrm{e}^{\sqrt{e^{\alpha p}} y / \ell}+\mathrm{e}^{-\sqrt{\mathrm{e}^{\alpha p}} y / \ell}}{2}\right) \\
& +B_{2}\left(\frac{\mathrm{e}^{\sqrt{\mathrm{e}^{\alpha p}} y / \ell}-\mathrm{e}^{-\sqrt{\mathrm{e}^{\alpha p}} y / \ell}}{2}\right)
\end{aligned}
$$

- Calculation values of the constants: $A, A_{1}, A^{\prime}, A_{1}^{\prime}$, $B_{1}, B_{2}, B_{1}^{\prime}$ and $B_{2}^{\prime}$

For $y=0$, Equation (A.10) becomes:

$$
\begin{array}{r}
u(0)=A_{0}+A_{1}(0)+\frac{1}{2 \mu_{0} \mathrm{e}^{\alpha p}} \frac{\partial p}{\partial x}(0)^{2}+B_{1} \operatorname{ch}\left(\sqrt{\mathrm{e}^{\alpha p}} 0 / \ell\right) \\
+B_{2} \operatorname{sh}\left(\sqrt{\mathrm{e}^{\alpha p}} 0 / \ell\right)
\end{array}
$$

With boundary conditions $(6 \div 9): u(x, 0, z)=u_{1}=0$, therefore, of Equation (A.12), we find that: $A_{0}=-B_{1}$.

we have :

$$
\begin{aligned}
\frac{\partial u}{\partial y}=A_{1}+\frac{1}{\mu_{0} \mathrm{e}^{\alpha p}} \frac{\partial p}{\partial x} y+ & B_{1} \frac{\sqrt{\mathrm{e}^{\alpha p}}}{\ell} \operatorname{sh}\left(\sqrt{\mathrm{e}^{\alpha p}} y / \ell\right) \\
& +B_{2} \frac{\sqrt{\mathrm{e}^{\alpha p}}}{\ell} \operatorname{ch}\left(\sqrt{\mathrm{e}^{\alpha p}} y / \ell\right)
\end{aligned}
$$

$$
\begin{aligned}
& \frac{\partial^{2} u}{\partial y^{2}}=\frac{1}{\mu_{0} \mathrm{e}^{\alpha p}} \frac{\partial p}{\partial x}+B_{1} \frac{\mathrm{e}^{\alpha p}}{\ell^{2}} \operatorname{ch}\left(\sqrt{\mathrm{e}^{\alpha p}} y / \ell\right) \\
&+B_{2} \frac{\mathrm{e}^{\alpha p}}{\ell^{2}} \operatorname{sh}\left(\sqrt{\mathrm{e}^{\alpha p}} y / \ell\right)
\end{aligned}
$$

while replacing, $y=0$, in Equation (A.14), we find that: $B_{1}=-\frac{\ell^{2}}{\mu_{0} \mathrm{e}^{2 \alpha p}} \frac{\partial p}{\partial x}$, then: $A_{0}=-B_{1}=\frac{\ell^{2}}{\mu_{0} \mathrm{e}^{2 \alpha p}} \frac{\partial p}{\partial x}$.

While putting, $A_{0}$ and $B_{1}$ in Equation (A.10), we obtain:

$$
\begin{aligned}
u(y) & =\frac{\ell^{2}}{\mu_{0} \mathrm{e}^{2 \alpha p}} \frac{\partial p}{\partial x}+A_{1} y+\frac{1}{2 \mu_{0} \mathrm{e}^{\alpha p}} \frac{\partial p}{\partial x} y^{2} \\
- & \frac{\ell^{2}}{\mu_{0} \mathrm{e}^{2 \alpha p}} \frac{\partial p}{\partial x} \operatorname{ch}\left(\sqrt{\mathrm{e}^{\alpha p}} y / \ell\right)+B_{2} \operatorname{sh}\left(\sqrt{\mathrm{e}^{\alpha p}} y / \ell\right)
\end{aligned}
$$

while replacing, $A_{0}$ and $B_{1}$ in Equation (A.14) we obtain:

$$
\begin{aligned}
\frac{\partial^{2} u}{\partial y^{2}}=\frac{1}{\mu_{0} \mathrm{e}^{\alpha p}} \frac{\partial p}{\partial x}-\frac{1}{\mu_{0} \mathrm{e}^{\alpha p}} & \frac{\partial p}{\partial x} \operatorname{ch}\left(\sqrt{\mathrm{e}^{\alpha p}} y / \ell\right) \\
& +B_{2} \frac{\mathrm{e}^{\alpha p}}{\ell^{2}} \operatorname{sh}\left(\sqrt{\mathrm{e}^{\alpha p}} y / \ell\right)
\end{aligned}
$$

For, $y=h$ we have, $\left.\frac{\partial^{2} u}{\partial y^{2}}\right|_{y=h}=0$, while replacing in Equation (A.16), we obtain:

$B_{2}=\frac{\ell^{2} \operatorname{ch}\left(\sqrt{\mathrm{e}^{\alpha p} h} / \ell\right)}{\mu_{0} \mathrm{e}^{2 \alpha p} \operatorname{sh}\left(\sqrt{\mathrm{e}^{\alpha p}} h / \ell\right)} \frac{\partial p}{\partial x}-\frac{\ell^{2}}{\mu_{0} \mathrm{e}^{2 \alpha p} \operatorname{sh}\left(\sqrt{\mathrm{e}^{\alpha p}} h / \ell\right)} \frac{\partial p}{\partial x}$

By replacing the value of $B_{2}$ in Equation (A.15):

$$
\begin{gathered}
u(y)=\frac{\ell^{2}}{\mu_{0} \mathrm{e}^{2 \alpha p}} \frac{\partial p}{\partial x}+A_{1} y+\frac{1}{2 \mu_{0} \mathrm{e}^{\alpha p}} \frac{\partial p}{\partial x} y^{2}-\frac{\ell^{2}}{\mu_{0} \mathrm{e}^{2 \alpha p}} \frac{\partial p}{\partial x} \operatorname{ch} \\
\times\left(\sqrt{\mathrm{e}^{\alpha p}} y / \ell\right)+\frac{\ell^{2} \operatorname{ch}\left(\sqrt{\mathrm{e}^{\alpha p}} h / \ell\right)}{\mu_{0} \mathrm{e}^{2 \alpha p} \operatorname{sh}\left(\sqrt{\mathrm{e}^{\alpha p}} h / \ell\right)} \operatorname{sh}\left(\sqrt{\mathrm{e}^{\alpha p} y} / \ell\right) \frac{\partial p}{\partial x} \\
-\frac{\ell^{2}}{\mu_{0} \mathrm{e}^{2 \alpha p} \operatorname{sh}\left(\sqrt{\mathrm{e}^{\alpha p}} h / \ell\right)} \operatorname{sh}\left(\sqrt{\mathrm{e}^{\alpha p}} y / \ell\right) \frac{\partial p}{\partial x}
\end{gathered}
$$

For, $y=h$ we have, $u(x, h, z)=U_{2}$, so Equation (A.17) becomes:

$$
U_{2}=A_{1} h+\frac{1}{2 \mu_{0} \mathrm{e}^{\alpha p}} \frac{\partial p}{\partial x} h^{2} \Rightarrow A_{1}=\frac{U_{2}}{h}-\frac{h}{2 \mu_{0} \mathrm{e}^{\alpha p}} \frac{\partial p}{\partial x}
$$

while replacing $A_{1}$ in Equation (A.17), we find:

$$
\begin{aligned}
u(y) & =\frac{U_{2}}{h} y \\
& +\frac{1}{2 \mu_{0} \mathrm{e}^{2 \alpha p}} \frac{\partial p}{\partial x}\left[\frac { y ( y - h ) } { \mathrm { e } ^ { - \alpha p } } 2 \ell ^ { 2 } \left(1+\frac{\operatorname{ch}\left(\sqrt{\mathrm{e}^{\alpha p} h} / \ell\right)}{\operatorname{sh}\left(\sqrt{\mathrm{e}^{\alpha p} h} / \ell\right)}\right.\right. \\
& \left.\left.\times \operatorname{sh}\left(\sqrt{\mathrm{e}^{\alpha p}} y / \ell\right)-\operatorname{ch}\left(\sqrt{\mathrm{e}^{\alpha p}} y / \ell\right)-\frac{\operatorname{sh}\left(\sqrt{\mathrm{e}^{\alpha p}} y / \ell\right)}{\operatorname{sh}\left(\sqrt{\mathrm{e}^{\alpha p}} h / \ell\right)}\right)\right]
\end{aligned}
$$

In the end, we find:

$$
\begin{aligned}
& u(y)=\frac{U_{2}}{h} y+\frac{1}{2 \mu_{0} \mathrm{e}^{2 \alpha p}} \frac{\partial p}{\partial x} \\
& \times\left[\frac{y(y-h)}{e^{-\alpha p}}+2 \ell^{2}\left(1-\frac{\operatorname{ch}\left(\frac{2 \sqrt{\mathrm{e}^{\alpha p}} y-\sqrt{\mathrm{e}^{\alpha p} h}}{2 l}\right)}{\operatorname{ch}\left(\frac{\sqrt{\mathrm{e}^{\alpha p}} h}{2 \ell}\right)}\right)\right]
\end{aligned}
$$


Same manner for $\mathbf{w}$ :

$$
\begin{aligned}
w(y)= & \frac{1}{2 \mu_{0} \mathrm{e}^{2 \alpha p}} \frac{\partial p}{\partial z} \\
& \times\left[\frac{y(y-h)}{e^{-\alpha p}}+2 \ell^{2}\left(1-\frac{\operatorname{ch}\left(\frac{2 \sqrt{\mathrm{e}^{\alpha p}} y-\sqrt{\mathrm{e}^{\alpha p}} h}{2 \ell}\right)}{\operatorname{ch}\left(\frac{\sqrt{\mathrm{e}^{\alpha p}} h}{2 \ell}\right)}\right)\right]
\end{aligned}
$$

finally, the fluid velocity components $u$ and $w$ for a piezoviscous couple stress fluid are:

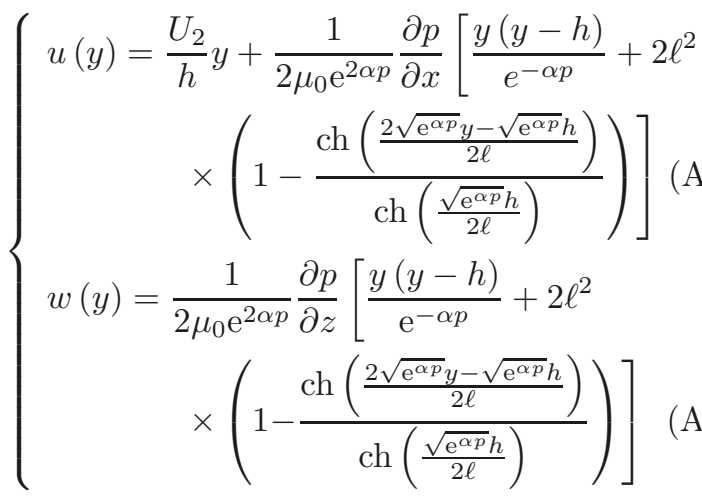

\section{References}

[1] V.K. Stokes, Couple stresses in fluids, Phys. Fluids 9 (1966) 1709-1715

[2] T.T. Ariman, N.D. Sylvester, Microcontinuum fluid mechanics, A review Int. J. Eng. Sci. 11 (1973) 905-930

[3] T.T. Ariman, N.D. Sylvester, Application of micro continuum fluid mechanics, J. Eng. Sci. 12 (1974) 273-293

[4] R.S. Gupta, L.G. Sharma, Analysis of couple stress lubricant in hydrostatic thrust bearing, Wear 125 (1988) $257-269$

[5] N.M. Bujurke, N.G. Naduvinamani, On the performance of narrow porous journal bearing lubricated with couple stress fluid, Acta Mech. 86 (1991) 179-191

[6] J.R. Lin, Static characteristics of rotor journal bearing system lubricated with couple stress fluids, Comput. Struct. 62 (1997) 175-184

[7] J.R. Lin, Squeeze film characteristics of long partial journal bearings lubricated with couple-stress fluids, Tribol. Int. 30 (1997) 53-58

[8] J.R. Lin, Effects of couple stresses on the lubrication of finite journal bearings, Wear 206 (1997) 171-178

[9] J.R. Lin, Squeeze film characteristics of finite journal bearings: couple-stress fluid model, Tribol. Int. 31 (1998) 201-207

[10] J.R. Lin, Static and dynamic characteristics of externally pressurized circular bearings lubricated with couplestress fluids, Tribol. Int. 32 (1999) 207-216

[11] J.R. Lin, Linear stability analysis of a rotor-bearing system: couple-stress fluid model, Comput. Struct. 79 (2000) 801-809
[12] A.A. Elsharkawy, Guedouar, An inverse method for finite journal bearings lubricated with couple stress fluids, Tribol. Int. 34 (2001) 107-118

[13] N.B. Naduvinamani, P.S. Hiremath, G. Gurubasavaraja, Surface roughness effects in a short porous journal bearing with a couple stress fluid, Fluid Dyn. Res. 31 ( 2002) 333-354

[14] Hsiu-Lu Chiang, Cheng-Hsing Hsu, Jaw-Ren Lin, Lubrication performance of finite journal bearings considering effects of couple stresses and surface roughness, Tribol. Int. 37 (2004) 297-307

[15] Yan-Yan Ma, Wei-Hua Wang, Xian-Hua Cheng, A study of dynamically loaded journal bearings lubricated with non-Newtonian couple stress fluids, Tribol. Lett. 17 (2004) 69-74

[16] M. Lahmar, Elasto-hydrodynamic analysis of doublelayered journal bearings lubricated by couple-stress fluids, J. Eng. Tribol. Proc. IMech E, Part J 219 (2005) $145-171$

[17] N.B. Naduvinamani, A. Siddangouda, Effect of surface roughness on the hydrodynamic lubrication of porous step-slider bearings with couple stress fluids, Tribol. Int. 40 (2006) 780-793

[18] Cai-Wan Chang-Jiana, Chao-Kuang Chen, Bifurcation analysis of flexible rotor supported by couple-stress fluid film bearings with non-linear suspension systems, Tribol. Int. 41 (2008) 367-386

[19] H. Boucherit, M. Lahmar, B. Bou-Saïd, Misalignment effects on steady-state and dynamic behaviour of compliant journal bearings lubricated with couple stress fluids, J. Lubrication Science John Wiley Editor 20 (2008) 241268

[20] H. Boucherit, M. Lahmar, B. Bou-Saïd, J. Tichy, Comparison of Non-Newtonian Constitutive Laws in Hydrodynamic Lubrication, Tribology Letters, James Lauer Memorial Issue, Guest Editor: Frank Talke 40 (2010) 49-57

[21] H. Boucherit, M. Lahmar, Étude théorique et numérique des effets combinés du comportement non-newtonien et de la piezoviscosité du lubrifiant sur les performances statiques d'un palier compliant, Matériaux \& Techniques 100 (2012) 87-100

[22] V. Petrone, A. Senatore, V. D'Agostino, Effect of an Improved Yasutomi Pressure-Viscosity Relationship on the Elastohydrodynamic Line Contact Problem, ISRN Tribology, 2013, Article ID 149451, 2013 (2013) 149451

[23] K.G. Binu, B.S. Shenoy, D.S. Rao, R. Pai, Static characteristics of a fluid film bearing with $\mathrm{TiO} 2$ based nanolubricant using the modified Krieger-Dougherty viscosity model and couple stress model, Tribol. Int. 75 (2014) 6979

[24] H. Van Leeuwen, The determination of the pressureviscosity coefficient of a lubricant through an accurate film thickness formula and accurate film thickness measurements, Proc. Inst. Mech. Eng. Part J: J. Eng. Tribol. 223 (2009) 1143-1163 
[25] V. D'Agostino, V. Petrone, A. Senatore, Effects of the Lubricant Piezo-Viscous Properties on EHL Line and Point Contact Problems, Tribol. Lett. 49 (2013) 385-396

[26] D. Nicolas, Les paliers hydrodynamiques soumis à un torseur de forces quelconque, Thèse de Docteur Ingénieur, INSA, Lyon, 1972

[27] C. Barus, Isotherms, isopiestics and isometrics relative to viscosity, Am. J. Sci. 45 (1893) 87-96
[28] D. Dowson, G.R. Higginson, Elasto-hydrodynamic lubrification, the fundamentals of roller and gear lubrication, Pergamon Press, Oxford, 1966

[29] E. Hoglund, Influence of lubricant properties on elastohydrodynamic, lubrication, Wear 232 (1999) 178-184

[30] A. Kabouya, M. Lahmar, B. Bou-Saïd, Étude des paliers lisses mésalignés lubrifiés par des fluides à couple de contrainte, Mécanique \& Industries 8 (2007) 577-595 